\title{
InstaQuest: aprender investigando. ¿Y por qué no?
}

\author{
InstaQuest: researching for \\ learning. And why not?
}

CARmen María Calama-GonzÁlez

https://orcid.org/0000-0002-6511-2885

Universidad de Sevilla. Departamento

Construcciones Arquitectónicas 1.

ccalama@us.es

DOI: http://dx.doi.org/10.12795/9788447231003.081

Pp.: 1736-1762 


\section{Contexto de la innovación}

El 14 de marzo de 2020 será recordado en los libros de historia como aquel en el que se decretó el estado de alarma para la gestión de la crisis sanitaria del COVID-19. Este hecho ha supuesto el inicio de un nuevo paradigma laboral, en el que el teletrabajo, las redes sociales y otras tecnologías de la comunicación y la información han adquirido una importancia vital.

En este panorama, la docencia ha requerido también una adaptación al mundo digital, que permitiera continuar la rutina educativa y los procesos formativos de los estudiantes, ayudando, a su vez, a hacer más llevadero el obligado confinamiento en el hogar.

La innovación didáctica planteada en el presente Ciclo de Innovación y Mejora en el Aula (CIMA) se aplica a la asignatura de "Acondicionamiento e Instalaciones 1", del Grado en Fundamentos en Arquitectura de la Universidad de Sevilla, a la que le corresponde un desarrollo semanal de cuatro horas (6 ETCS). Esta asignatura cuenta con una media de unos 25 estudiantes por clase y los contenidos que se imparten están vinculados con el diseño, dimensionado e integración en edificios residenciales y terciarios de varias instalaciones: protección contra incendios, abastecimiento de agua, agua caliente sanitaria, saneamiento, ventilación, gases combustibles y telecomunicaciones. El objetivo final consiste en el desarrollo de una serie de competencias que permitan al estudiante adquirir los conocimientos necesarios para la resolución de problemas reales en su futuro profesional, a partir de: la interpretación de datos, el análisis y la síntesis, la organización y planificación de tareas; el razonamiento lógico y la valoración crítica de posibles soluciones, potenciando la creatividad, la visión espacial y la habilidad gráfica general; un compromiso ético, a partir de la concienciación de

Ciclos de Mejora en el Aula (2020). Experiencias de Innovación Docente de la US Esta obra se distribuye con la licencia Creative Commons 
condicionantes económicos, normativos y deontológicos, y una sensibilidad hacia temas medioambientales; o la valoración del trabajo en equipo, entre otros aspectos.

Se parte de la experiencia del curso previo, durante el cual se aplicó un CIMA a otra unidad didáctica (Calama-González, 2020). La buena acogida por parte del alumnado y las conclusiones, tanto positivas como negativas, son el motor del presente trabajo Entre las conclusiones, hay que destacar que se logró potenciar el razonamiento de los estudiantes y el debate colectivo, mediante el fomento de las tareas prácticas y la reducción del contenido teórico, diferenciando entre teoría de primer nivel (conceptos generales impartidos durante las sesiones teóricas) y de segundo (conceptos específicos y complementarios transmitidos durante las sesiones prácticas). Como parte negativa, se ha de mencionar el querer abarcar una larga lista de actividades de mejora en un tiempo bastante limitado, lo que conllevó a tener que acortar el tiempo dedicado a algunas tareas, dado que la planificación que se realizó no fue la más adecuada. Otro aspecto relevante del curso anterior fue el disponer de docencia compartida con otro profesor, lo que en cierta medida limitaba la capacidad de innovación e incrementaba la problemática del desarrollo del CIMA en un determinado contexto temporal.

Entre las dificultades de aprendizaje detectadas, cobra relevancia el hecho de que esta asignatura es el primer contacto que tiene el alumnado con las instalaciones de los edificios, por lo que es primordial aprender una serie de conceptos básicos para su posterior aplicación práctica. Pero dichos conceptos se convierten en obstáculos para el aprendizaje, dado su elevada carga técnica y normativa. A ello se suma, la preferencia del alumnado por otras asignaturas más ligadas al diseño de proyectos arquitectónicos, donde la subjetividad, creatividad e inventiva se desarrollan con mayor libertad. Por ello, motivar al

Ciclos de Mejora en el Aula (2020). Experiencias de Innovación Docente de la US Esta obra se distribuye con la licencia Creative Commons 
alumnado para que se interese por la asignatura y mantener su atención se convierten en una tarea ardua y de gran complejidad.

Otro obstáculo para este CIMA es la presión de tener un contexto docente común para toda la asignatura en los diferentes grupos de clase, compartiendo los mismos recursos, tanto docentes como estudiantes, a través de la Plataforma Virtual de la Universidad de Sevilla (temas de la asignatura, normativas, ejercicios prácticos...). Esto implica que la evaluación de la asignatura es igualmente común, limitando la posibilidad de incorporar estrategias de mejora más allá de en la propia metodología de enseñanza, es decir, los contenidos y criterios de evaluación deben mantenerse para la adecuada coordinación entre el profesorado de la asignatura. Afortunadamente, este curso se dispone de docencia completa en el grupo en el que se va a aplicar el CIMA, lo que en cierta medida flexibiliza las posibles actuaciones de mejora.

La experimentación docente de este CIMA tiene por objeto la mejora del proceso de enseñanza y aprendizaje a distancia de la unidad didáctica de Gases Combustibles, a la que le corresponde un desarrollo temporal de 8 horas ( 4 horas teóricas y 4 horas prácticas).

\section{Objetivos y principios didácticos}

En base a lo anterior, se pretende alcanzar los siguientes objetivos didácticos:

- Fomentar la participación activa del alumnado, potenciando su motivación intrínseca para despertar su interés y su aprendizaje autónomo.

- Apostar por la cooperación y colaboración en equipos de trabajo, el intercambio fructífero de ideas, el

Ciclos de Mejora en el Aula (2020). Experiencias de Innovación Docente de la US Esta obra se distribuye con la licencia Creative Commons 
razonamiento crítico-reflexivo o la puesta en común de resultados.

- Promover el desarrollo de competencias en el alumnado, incentivando su pensamiento analítico, crítico, sistémico, práctico y creativo para la resolución de problemas reales.

- Incrementar las actividades prácticas para crear sesiones más interactivas, donde los estudiantes sean los protagonistas y la profesora adquiera el papel de moderadora y guía.

- Promover prácticas docentes para la mejora continua del proceso de enseñanza-aprendizaje, derivando en un impacto positivo en el rendimiento académico del alumno.

Para el adecuado diseño y planificación del CIMA, se han considerado los siguientes:

- Identificar las ideas previas del alumnado a través del planteamiento de una disonancia cognitiva (Piaget, 1981) permitirá valorar si es necesario un ajuste de la metodología docente, para que los estudiantes alcancen el equilibrio conceptual. Su contextualización en una situación cotidiana o actual, permitirá crear un entorno en el que el alumnado se interese por la materia (motivación intrínseca).

- Fomentar el trabajo cooperativo y colaborativo en equipos: la retroalimentación entre el alumnado, valorando el factor social durante el aprendizaje. Esto se refiere a la Zona de Desarrollo Próximo (Vygotsky , 2012) o la distancia entre el desarrollo efectivo del alumno (lo que es capaz de hacer de forma autónoma) y el nivel de desarrollo potencial (lo que es capaz de hacer con la ayuda de sus compañeros y/o docente).

- Potenciar el aprendizaje autónomo como estrategia para el desarrollo de la competencia de aprender a aprender y como autorregulación del estudiante, es decir, la 
planificación de tareas, supervisión de su desarrollo y evaluación de las mismas.

- Aprender por descubrimiento (Bruner, 2011) para resolver problemas o casos prácticos reales que requieren una investigación activa personal o grupal. Con este enfoque, el aprendizaje trasciende del tradicional deductivo (propio de las clases transmisivas) o memorístico (aprender por repetición), hacia el aprendizaje significativo (Ausubel, 1976): el estudiante asocia la nueva información con las ideas previas, asimila conceptos y adquiere el conocimiento necesario para su aplicación efectiva a una situación práctica.

- Guiar el proceso de aprendizaje del alumnado en base a la Teoría del Andamiaje (Guilar, 2009). La profesora cede el protagonismo al alumnado y les aporta los recursos necesarios para que sean capaces de construir su propio conocimiento, retirando los andamiajes o sustituyéndolos por otros a medida que se avance en el aprendizaje.

- Motivar de forma extrínseca. Pese a que la motivación debe proceder fundamentalmente del propio estudiante (intrínseca), su combinación con actuaciones que permitan una motivación extrínseca le permitirá ser consciente de que se valora su esfuerzo. Con ello, se pone el énfasis en la autoconfianza o autosatisfacción ante el éxito en la resolución de problemas (Gagné, 1987). Para promover la motivación extrínseca se emplean refuerzos positivos que tengan un impacto en la evaluación del alumnado, de tal forma que se valore el esfuerzo del alumno y el tiempo que ha dedicado.

\section{Diseño previo del CIMA}

El modelo metodológico tradicional de la asignatura (Figura 1) consiste en la división de cada sesión semanal en dos partes (cada una de dos horas): tras la impartición de una base teórica en la que todos los recursos

Ciclos de Mejora en el Aula (2020). Experiencias de Innovación Docente de la US Esta obra se distribuye con la licencia Creative Commons 
materiales (contenidos temáticos y normativas) se facilitan de forma previa al alumnado, se pide a los alumnos que trasladen esos conocimientos a la práctica mediante el diseño, cálculo e integración de esa instalación en un edificio real, cuya planimetría y datos se les facilitan. La evaluación final del curso, incluida en la programación docente de la asignatura, consta de: $60 \%$ de la actividad práctica grupal, $30 \%$ de una prueba de conocimientos teórico-prácticos y $10 \%$ de asistencia y participación en los

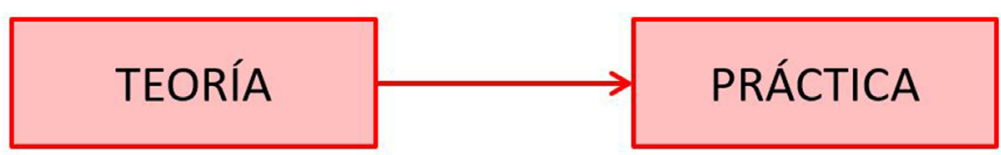

Figura 1. Modelo metodológico tradicional

Con el nuevo modelo metodológico planteado (Figura 2), se enfatiza la participación activa del alumnado en su proceso de aprendizaje y su vinculación más directa con los procedimientos de trabajo propios de la práctica profesional. Se parte de una evaluación previa de los conocimientos del alumnado para el adecuado ajuste y organización de la práctica docente (?). A continuación, se plantea al alumnado una experiencia de investigación basada en una actividad contextualizada en su realidad próxima, que genere interacciones proactivas dentro del grupo de trabajo y en la clase. El alumnado va adquiriendo de forma autónoma ciertos conocimientos que debe intentar aplicar a la resolución del caso práctico, siendo guiado por la profesora, quien completa aquellos conceptos que el alumnado no sea capaz de alcanzar de forma autónoma o con sus compañeros (conceptos estructurales de mayor importancia). Seguidamente, en base a la nueva información, se produce un feedback alumnado-profesora y alumnado-alumnado que permite retomar el proceso de investigación y de aplicación práctica con una visión más nutrida y completa, incorporando conceptos más específicos.

Ciclos de Mejora en el Aula (2020). Experiencias de Innovación Docente de la US Esta obra se distribuye con la licencia Creative Commons 


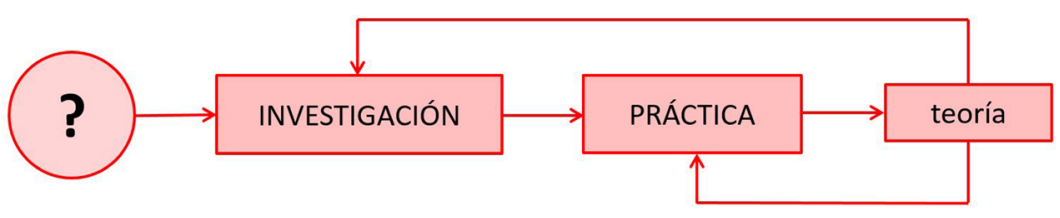

Figura 2. Modelo metodológico diseñado (docencia presencial)

Esta metodología requiere la jerarquización de los contenidos de la asignatura de mayor a menor importancia (primer y segundo nivel), diferenciando entre conceptos declarativos, procedimentales y actitudinales, además de los contenidos transversales a la materia. Con todo esto, se programa y planifica una secuencia de actividades, teniendo en cuenta el contexto del alumnado, los recursos disponibles y la temporalización de la unidad, entre otros aspectos.

Para la docencia a distancia, este modelo se ha adaptado (Figura 3) conforme a los recursos disponibles en la Plataforma Virtual de la Universidad de Sevilla, en concreto, la herramienta Blackboard Collaborate Ultra. Esta aplicación permite la impartición de docencia de forma no presencial, mediante la conexión de diversos dispositivos (móvil, Tablet, ordenadores...) para mantener sesiones tipo webinar, donde los participantes pueden interactuar, produciéndose una retroalimentación constante, cara a cara, voz a voz o compartiendo recursos en tiempo real. Además, facilita la realización de labores de tutorización, dando la posibilidad de crear sesiones personalizadas o grupales.

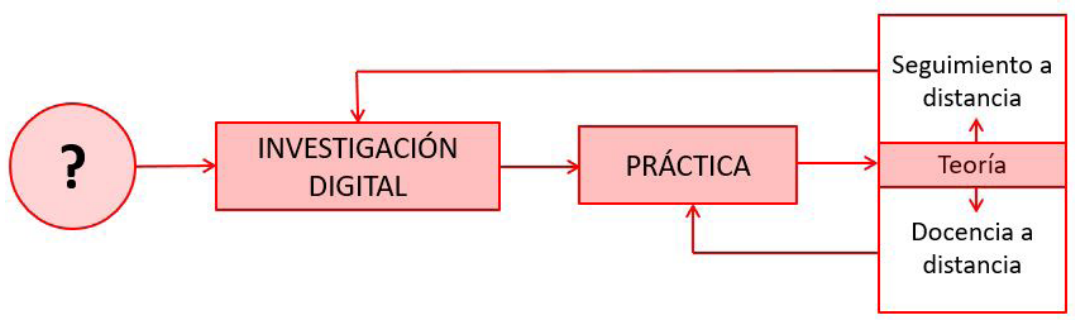

Figura 3. Modelo metodológico propuesto adaptado (docencia virtual)

Ciclos de Mejora en el Aula (2020). Experiencias de Innovación Docente de la US Esta obra se distribuye con la licencia Creative Commons 
Todas las actividades planteadas en este CIMA, se han desarrollado en el plano virtual, mediante el trabajo colaborativo y cooperativo a través de la interconexión digital, potenciando, de esta forma, el empleo adecuado de recursos digitales y el manejo de páginas web oficiales.

\section{Mapa de contenidos y secuencias de actividades}

En la unidad seleccionada el alumnado aprende cómo funciona, se diseña e integra una instalación de gas en un edificio. Los contenidos a impartir durante el CIMA se han indicado en un mapa conceptual (Figura 4), distinguiendo entre los de primer nivel (conceptos generales, básicos y necesarios para desarrollar conceptos posteriores) y de segundo nivel (conceptos de profundización, más específicos y técnicos). El mapa debe leerse en el sentido de las flechas, reflejando el orden metodológico lógico a seguir en el aprendizaje de conceptos (de menor a mayor complejidad) para llevar a cabo un adecuado diseño y dimensionado de la instalación, quedando patente la importancia de la interconexión entre los elementos que integran este sistema. En el mapa se han indicado en color azul los contenidos declarativos, en rojo los procedimentales y en verde los aptitudinales. El grado de profundización en los mismos, refleja su importancia. La fuente normal se ha empleado para representar los contenidos de primer nivel (generales) y, en negrita, se indican los contenidos de segundo nivel (específicos).

Los contenidos seleccionados se organizan en una serie de actividades a desarrollar en las sesiones teórico-prácticas, indicadas en el mapa de contenidos (A1 a A7). En la Tabla 1 se muestra el cuestionario inicial elaborado para la Actividad 2 (A2), aclarando en las instrucciones que es totalmente anónimo y su cumplimentación se valorará positivamente en la calificación final. Presenta cinco preguntas que deberán resolverse de forma individual tras la

Ciclos de Mejora en el Aula (2020). Experiencias de Innovación Docente de la US Esta obra se distribuye con la licencia Creative Commons 
lectura de dos noticias sobre el tema, publicadas en la prensa digital. Este cuestionario se emplea también al final de la unidad para evaluar el proceso de aprendizaje de cada alumno. La Tabla 2 indica la temporalización de las actividades planteadas, así como los materiales y recursos necesarios para su realización. Esta secuenciación se ha realizado en base a la adecuación psicopedagógica del alumnado, la progresión en el grado de complejidad, las conexiones interdisciplinares y la educación en valores y temáticas transversales. Es preciso indicar que se trabaja con un grupo de 24 estudiantes, de entre los cuales, 16 están cursando por segunda vez esta asignatura. Los equipos de trabajo deberán incluir tanto alumnos repetidores como estudiantes que cursan esta asignatura por primera vez, creando grupos heterogéneos y mixtos.

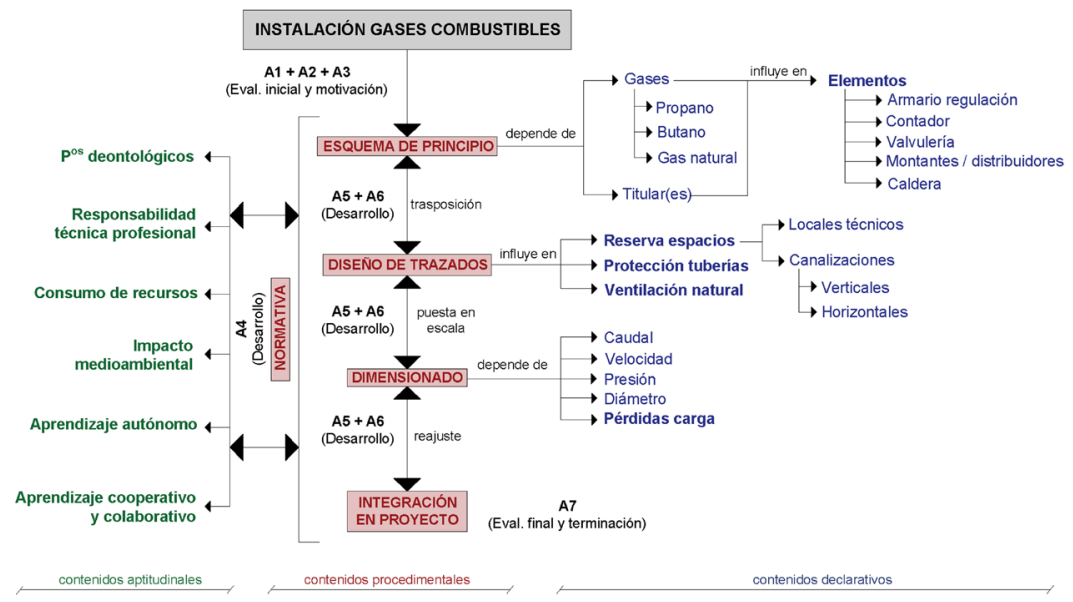

Figura 4. Mapa conceptual: conceptos aptitudinales (verde), procedimentales (rojo) y declarativos (azul). Actividades (A1-A7). Nivel profundización: bajo (normal), alto (negrita).

Ciclos de Mejora en el Aula (2020). Experiencias de Innovación Docente de la US Esta obra se distribuye con la licencia Creative Commons 
Tabla 1. Cuestionario inicial y final. A1 (I y II) de la secuencia de actividades.

A1_CUESTIONARIO

INICIAL GASES COMBUSTIBLES

\section{Alias:}

Instrucciones: Este cuestionario se entrega antes de iniciar la unidad didáctica para valorar las ideas previas que tienes y enfocar el proceso de aprendizaje de la forma más adecuada posible. Si desconoces la respuesta a alguna de las preguntas, indica "No lo sé" o, mejor, utiliza tu pensamiento creativo y lógico para responder. Cuando termines, argumenta tus respuestas en tu grupo de la Blackboard. A final se hará una puesta en común en la clase.

Este cuestionario NO ES EVALUABLE y es TOTALMENTE ANÓNIMO, pero te agradecería que escribieras un Alias, el que más te guste, ya que al final de la impartición del tema se entregará un cuestionario final, para poder evaluar tu progreso de aprendizaje. Por tanto, el tiempo y esfuerzo que dediques a completar este cuestionario, se VALORARÁ POSITIVAMENTE, influyendo en la calificación final de la asignatura.

¿Qué tienes que hacer?: Busca las siguientes noticias y contesta a las preguntas.

"Tres afectados en el incendio de una vivienda provocado por una deflagración de gas en Marbella". (04/03/2020).

"Evacuado al hospital un herido tras una explosión de gas butano en su vivienda de Algeciras (Cádiz)". (29/12/2019)

1. ¿Cuáles crees que pueden ser los peligros asociados a una instalación de gas en un edificio? Justifícalo.

2. ¿Qué medidas de seguridad adoptarías para minimizar esos riesgos? Justificalo.

3. ¿Qué gases combustibles conoces que se empleen en los edificios? Menciona algunas de sus propiedades..

4. ¿Qué posibles aplicaciones puede tener una instalación de gas en un edificio? Justificalo.

5. Según los gases que has nombrado, ¿qué reserva de espacios tendrías en cuenta en un edificio? Justificalo.

El ejercicio práctico a investigar y resolver por el alumnado es un InstaQuest: similar a un WebQuest pero empleando los recursos digitales que ofrece la Universidad de Sevilla (Tabla 3): a medida que resuelvan un apartado, se desbloqueará el siguiente. Para resolver un apartado, los estudiantes deberán utilizar sus habilidades de Ciclos de Mejora en el Aula (2020). Experiencias de Innovación Docente de la US
Esta obra se distribuye con la licencia Creative Commons 
investigación y de consulta de recursos digitales, potenciando así el aprendizaje por descubrimiento.

\section{Tabla 2. Secuencia de actividades}

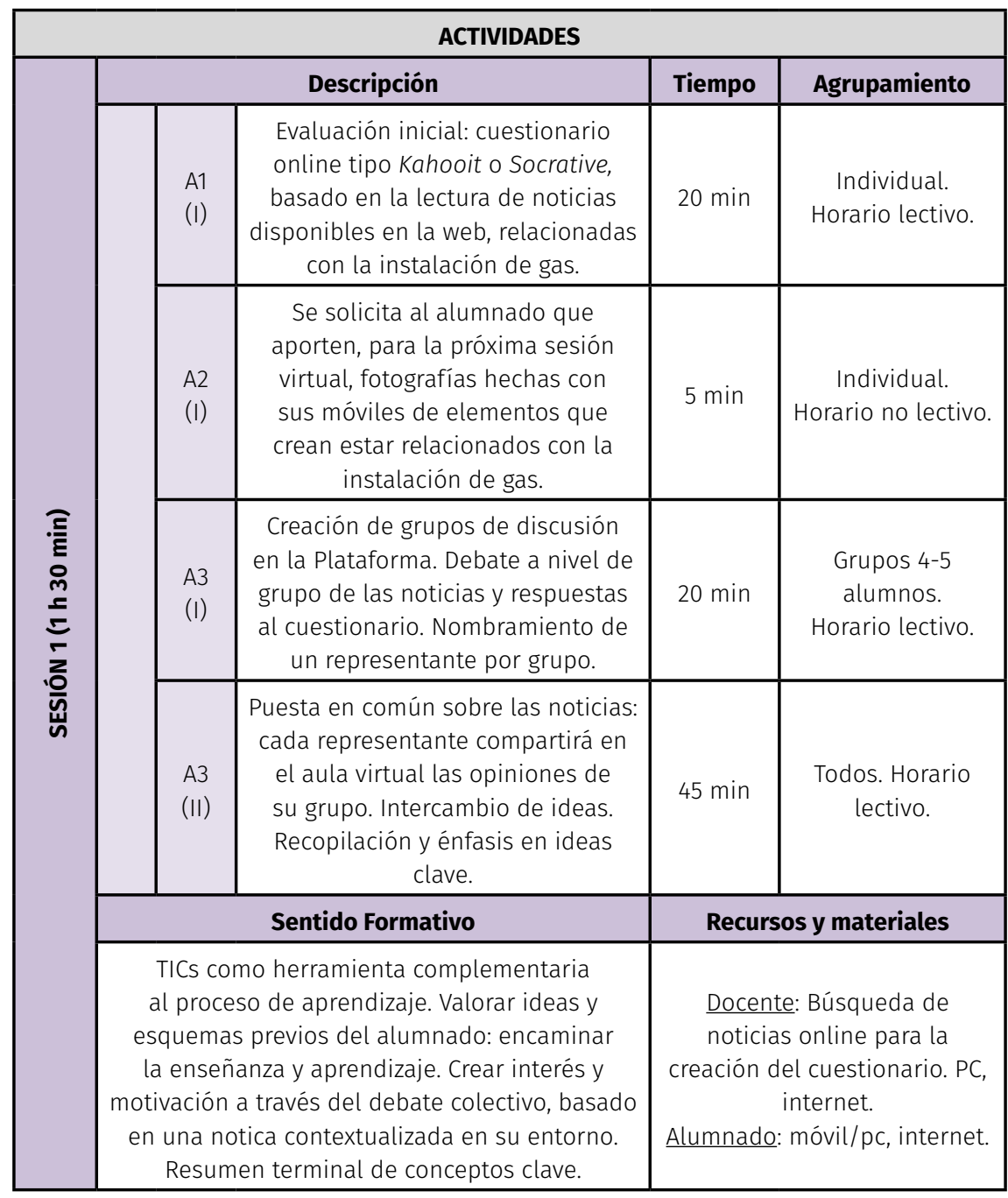

Ciclos de Mejora en el Aula (2020). Experiencias de Innovación Docente de la US Esta obra se distribuye con la licencia Creative Commons 


\begin{tabular}{|c|c|c|c|c|c|}
\hline \multirow{8}{*}{ 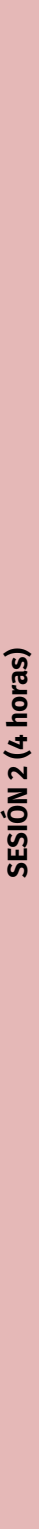 } & \multicolumn{3}{|r|}{ Descripción } & Tiempo & Agrupamiento \\
\hline & \multirow{5}{*}{ 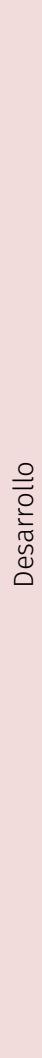 } & $\begin{array}{l}\mathrm{A} 2 \\
(\mathrm{II})\end{array}$ & $\begin{array}{c}\text { Puesta en común de las fotografías } \\
\text { en el aula virtual: cada alumno } \\
\text { explicará la imagen aportada y } \\
\text { su relación con la instalación. } \\
\text { Recopilación y énfasis en ideas } \\
\text { clave. }\end{array}$ & $30 \mathrm{~min}$ & $\begin{array}{c}\text { Todos. Horario } \\
\text { lectivo. }\end{array}$ \\
\hline & & A4 & $\begin{array}{l}\text { Explicación del docente al } \\
\text { alumnado del ejercicio práctico } \\
\text { InstaQuest. }\end{array}$ & $10 \mathrm{~min}$ & $\begin{array}{c}\text { Todos. Horario } \\
\text { lectivo. }\end{array}$ \\
\hline & & $\begin{array}{l}\text { A5 } \\
(\mathrm{I})\end{array}$ & $\begin{array}{l}\text { Envío al alumnado del ejercicio } \\
\text { práctico InstaQuest: el alumnado, } \\
\text { a través de los grupos de la } \\
\text { Plataforma, deberá investigar sobre } \\
\text { la práctica con los enlaces web } \\
\text { facilitados, vinculados con recursos } \\
\text { normativos, textos oficiales y } \\
\text { noticias trabajadas, para completar } \\
\text { el contenido del ejercicio. }\end{array}$ & $80 \mathrm{~min}$ & $\begin{array}{l}\text { Grupos 4-5 } \\
\text { alumnos. } \\
\text { Horario lectivo. }\end{array}$ \\
\hline & & $\begin{array}{l}\text { A6 } \\
(I)\end{array}$ & $\begin{array}{l}\text { Sesión teórica en la Plataforma: } \\
\text { conceptos de primer nivel y análisis } \\
\text { de edificios reales. Recopilación y } \\
\text { énfasis en ideas clave. }\end{array}$ & $60 \mathrm{~min}$ & $\begin{array}{c}\text { Todos. Horario } \\
\text { lectivo. }\end{array}$ \\
\hline & & $\begin{array}{l}\text { A5 } \\
(I I)\end{array}$ & $\begin{array}{l}\text { Continuación del desarrollo del } \\
\text { ejercicio práctico, tras los nuevos } \\
\text { conocimientos adquiridos en la } \\
\text { sesión teórica. }\end{array}$ & $60 \mathrm{~min}$ & $\begin{array}{l}\text { Grupos 4-5 } \\
\text { alumnos. } \\
\text { Horario lectivo. }\end{array}$ \\
\hline & & & Sentido Formativo & \multicolumn{2}{|c|}{ Recursos y materiales } \\
\hline & \multicolumn{3}{|c|}{$\begin{array}{l}\text { Concienciar sobre la importancia de los textos } \\
\text { legislativos en la práctica profesional. Dosificar } \\
\text { el contenido teórico para no abrumar al } \\
\text { alumnado: diferenciación entre conceptos de } \\
\text { primer y segundo nivel. Potenciar el aprendizaje } \\
\text { por descubrimiento y el trabajo cooperativo y } \\
\text { colaborativo. Fomentar la autonomía (aprender } \\
\text { a aprender). Desarrollar el pensamiento } \\
\text { analítico, crítico, lógico y sistémico. }\end{array}$} & \multicolumn{2}{|c|}{$\begin{array}{l}\text { Docente: Ejercicio práctico } \\
\text { InstaQuest. Textos normativos. } \\
\text { Material teórico, pc, internet: } \\
\text { multimedia, esquemas. } \\
\text { Alumnado: móvil/pc, internet. }\end{array}$} \\
\hline
\end{tabular}

Ciclos de Mejora en el Aula (2020). Experiencias de Innovación Docente de la US Esta obra se distribuye con la licencia Creative Commons Reconocimiento-NoComercial-SinObraDerivada Internacional (CC BY-NC-ND 4.0.) 


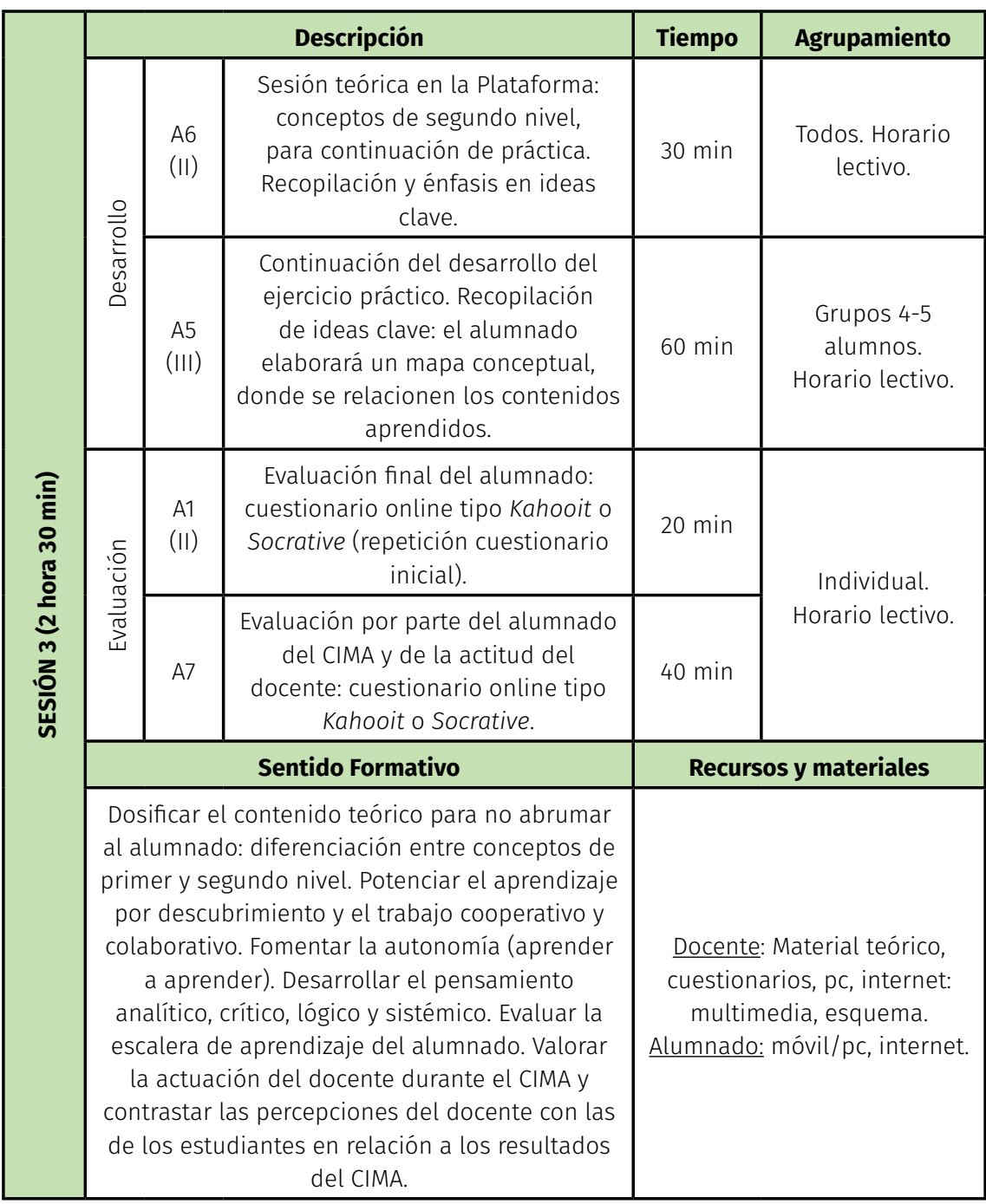

Ciclos de Mejora en el Aula (2020). Experiencias de Innovación Docente de la US Esta obra se distribuye con la licencia Creative Commons 
Tabla 3. Muestra del contenido del documento InstaQuest (A5 de la secuencia de actividades).

\section{A5_INSTAQUEST GASES COMBUSTIBLES}

A) Completa el esquema de principio. Observa el esquema de principio de una red de gas natural de un edificio residencial plurifamiliar y contesta a las siguientes preguntas, basándote en las pistas y utilizando tus habilidades de investigación. Deberás localizar las respuestas en el Manual de Instalaciones Receptoras de Gas Natural.

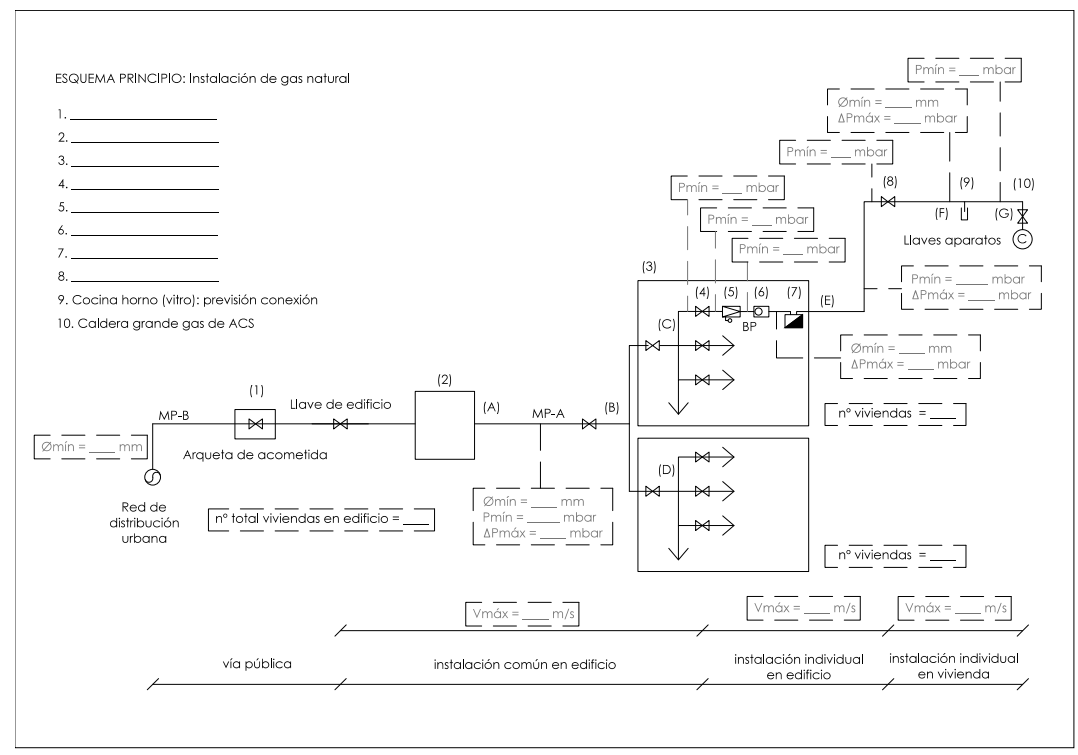

A1) El esquema de una instalación de gas natural tiene muchas similitudes con la red de fontanería, instalación que ya conoces. La conexión entre la red pública urbana y la red del edificio, se realiza mediante una arqueta ubicada en el acerado público, accesible por la compañía suministradora para su mantenimiento.

PREGUNTA 1. ¿Cómo se llama el elemento que alberga esta arqueta en su interior? Rellena el campo 1.
Pista: Consulta el Esquema MP-B en el Capítulo 4. Cálculo de instalaciones receptoras, Sección 4.2 Pérdidas de carga admisibles y diámetros mínimos.

Ciclos de Mejora en el Aula (2020). Experiencias de Innovación Docente de la US Esta obra se distribuye con la licencia Creative Commons Reconocimiento-NoComercial-SinObraDerivada Internacional (CC BY-NC-ND 4.0.) 
A2) En la instalación de gas natural, la red urbana y la parte común de la instalación del edifico, suelen proyectarse, respectivamente, a media presión B (MP-B), entre 0.4-4 bar efectivos, y a media presión A (MP-A), entre 0.05-0.4 bar efectivos. Esto se hace para reducir las pérdidas de presión en el transporte del gas. Sin embargo, en el interior de las viviendas, se trabaja a baja presión (BP), inferior a 0.05 bar, por criterios de seguridad.

PREGUNTA 2. Para reducir de MP-B a MP-A, ¿qué elemento se necesita instalar? Rellena el campo 2 .

PREGUNTA 3: Y para reducir de MP-A a BP, ¿qué elemento se instala? Rellena el campo 5.

Pista: Consulta el capítulo 1. Generalidades, en la sección 1.3 Terminología.

A3) Como en fontanería, la instalación de gas natural debe permitir el control del consumo de forma individual de cada titular de las viviendas que integran el edificio residencial (piensa en quién paga la factura del consumo de gas).

PREGUNTA 4. ¿Qué elementos permiten el control del consumo de gas? (campo 7). ¿Cómo se denominan esos elementos cuando se instalan en conjunto? (campo 3).

Pista: Consulta el esquema de la instalación del Módulo 2, tras la Introducción.

PREGUNTA 5. ¿Dónde se deben instalar en el edificio? ¿Es posible hacer paquetes de estos elementos e instalarlos en varios grupos? ¿Qué requisitos de seguridad se deben tener en cuenta para ello?

PREGUNTA 6. Completa los otros elementos de la instalación común e individual, hasta el montante de gas indicado en el esquema (campos 4 y 6). El elemento 6 permite a la compañía suministradora controlar que el usuario no sobrepasa el caudal de consumo contratado.

Pista: Consulta los esquemas del capítulo 3. Diseño y construcción en las pp. 3.2-6 a 3.2-11.

Pista: Consulta el esquema de Instalaciones receptoras en fincas plurifamiliares, del Capítulo 2. Esquemas tipo de instalaciones receptoras, en la sección 2.1 Instalaciones receptoras conectadas a redes en media presión $B$

A4) Como sabes, el diseño de los trazados horizontales y verticales de las instalaciones se hace, preferentemente, por zonas comunes, para que puedan ser accesibles para su mantenimiento, limpieza y reparación. Por ello, es importante encontrar una vertical en el proyecto, donde podamos ubicar los montantes de la instalación de gas, conectando así los elementos de la red comunitaria a nivel de edifico, con los de la red individual a nivel de vivienda.

PREGUNTA 7: ¿Qué criterios de diseño debes

considerar en el trazado de tuberías, para reducir al mínimo el riesgo ligado con la instalación de gases combustibles en los edificios? ¿Qué ventajas tiene el trazado de tuberías de gas por el exterior del edifico frente

Pista: Consulta los esquemas del capítulo 3. Diseño y construcción en las pp. 3.2-1 a 3.2-3. a por el interior? 
PREGUNTA 8: ¿Cuál es el primer elemento que se instala en el interior de la vivienda? Rellena el campo 8.
Pista: Consulta el esquema indicado en la pista de la pregunta 6.

B) Elabora el esquema de principio del ejercicio práctico. Para ello, adapta el esquema facilitado.

C) Diseña el trazado de la red comunitaria e interior en el ejercicio práctico.

D) Dimensionado de la red en el ejercicio práctico. Guíate por los siguientes comentarios.

- El dimensionado de la instalación de gas natural en un edifico tiene como objetivo el cálculo del diámetro $(\varnothing)$ de las tuberías de la red, debiendo realizarse por tramos.

- Para ello, es preciso determinar el caudal de cálculo (Q) que se debe considerar en cada tramo, la longitud del tramo (L) y fijar las pérdidas de carga máximas $\left(\Delta \mathrm{P}_{\text {max }}\right)$, como un valor límite indicado por la norma (dependiente del tramo considerádo). Determinado el diámetro de cálculo $(\phi)$, deberemos realizar varias comprobaciones:

- El diámetro nominal $\left(\phi_{n}\right)$ debe ser igual o mayor al de cálculo $\left(\phi_{c}\right)$ : $\phi_{\mathbf{n}} \emptyset_{\mathbf{c}}$

- El diámetro nominal $\left(\phi_{\mathrm{n}}\right)$ debe ser como mínimo $\left(\phi_{\text {min. }}\right)$ el indicado por la norma: $\phi_{n} \phi_{\text {min }}$

Comprueba que, con ese diámetro, cumples con la presión mínima $\left(\mathrm{P}_{\min }.\right)$, pérdida de carga real $(\Delta P)$ y velocidad máxima $\left(V_{\text {max }}\right)$, indicadas por la norma para ese tramo. Si no, deberás modificar el diámetro, de forma acorde.

PREGUNTA 9: Completa en el esquema de principio los valores límite indicados por la norma: pérdidas de carga máximas ( $\triangle$ Pmáx), presión mínima (Pmín.), velocidad máxima (Vmáx.) y el diámetro mínimo (Ømín.).
Pista: Consulta los esquemas indicados en las pistas de la pregunta 1 y 6 .

D1) Dimensiona, por tramos, el diámetro de la red de gas natural, utilizando la hoja de cálculo disponible en la Plataforma. Calcula la tubería más desfavorable (mayor longitud desde el último punto de consumo en la vivienda hasta el armario de regulación). Fijate en los tramos indicados en el esquema facilitado y define los tramos en el tuyo.

Ciclos de Mejora en el Aula (2020). Experiencias de Innovación Docente de la US Esta obra se distribuye con la licencia Creative Commons 
- Calcula el caudal de cada tramo. Considera que la instalación de gas en la vivienda incluye solo dos aparatos: una cocina horno (como previsión de la instalación de una vitro de gas) y una caldera grande para ACS.

- Determina el caudal nominal ( $\left.q_{i}\right)$ de cada aparato a instalar, según el Capítulo 4. Cálculo de instalaciones receptoras, en la sección 4.1 Datos básicos para el cálculo de instalaciones (p. 4.1-2).

- Determina el caudal máximo de simultaneidad total $\left(Q_{\text {, }}\right.$ ) en la vivienda, como el sumatorio del caudal nominal $\left(q_{i}\right)$ unitario de cada aparato $\left(q_{i}\right): \mathbf{Q}_{\mathbf{t}}=\mathbf{q}_{\mathbf{i}_{\text {_cocina-horno }}}$ $+\mathbf{q}_{\mathbf{i}_{\text {caldera }}}$

- Nota: Si fueran más de dos aparatos, a partir del tercero y para los de menor caudal, se debería considerar la mitad de su caudal (p. 4.1-3).

- Para calcular el caudal máximo de simultaneidad total de varias viviendas $\left(Q_{-}\right)$ multiplica el caudal máximo de simultaneidad total ( $\left.Q_{t}\right)$ en la vivienda por et número de viviendas, considerando un coeficiente de simultaneidad de uso (S): $\mathbf{Q}_{\mathrm{T}}=\mathbf{Q}_{\mathrm{t}} \times \mathbf{n}$ 으 viviendas $\times \mathbf{S}$

- El coeficiente de simultaneidad (S) depende del número de viviendas. Para este ejercicio, emplea S para cuando no existe caldera de calefacción. Consulta la tabla del Capítulo 4. Cálculo de instalaciones receptoras, en la sección 4.1 Datos básicos para el cálculo de instalaciones (p. 4.1-3)

- Si el número de viviendas (N) que consideras no está incluido en la tabla anterior, calcula el coeficiente de simultaneidad (S) a partir de la siguiente fórmula: $\mathbf{S}=\mathbf{( 1 9 + N )} /[\mathbf{1 0} \times(\mathbf{N}+\mathbf{1})]$

- Ten presente que el tramo AB del esquema facilitado se dimensiona con el total de viviendas en el edificio. En cambio, si hay varias centralizaciones de contadores, como sucede en los tramos BC y BD del esquema, el número de viviendas deberá ser coherente con el número de contadores en cada tramo.

Determina la longitud real $\left(L_{2}\right)$ de cada tramo que estés dimensionando. En la hoja de cálculo, obtendrás la longitud equivalente $\left(L_{e}\right)$ del tramo. $L_{e}$ tiene en cuenta un incremento del $20 \%$ de $\mathrm{L}$ con vistas a incluir en ${ }^{2}$ el cálculo la consideración de las pérdidas de carga en la tubería (pérdidas de presión por rozamiento).

- Fija las pérdidas de carga máximas $\left(\Delta P_{\text {máx }}\right)$ de la red en función del tramo que estés calculando: guíate de los valores que has completado en el esquema de principio facilitado.

- Con ello, en la hoja obtendrás, para cada tramo incluido, el diámetro de cálculo $\left(\phi_{c}\right)$. Selecciona el diámetro nominal $\left(\phi_{n}\right)$, cumpliendo con el mínimo de la norma y con lo explicado en D.

- Por último, comprueba el cumplimiento a pérdidas de carga máximas $\left(\Delta \mathrm{P}_{\text {máx }}\right)$, presión mínima $\left(P_{\text {min }}\right)$ y velocidad máxima $\left(V_{\text {máx }}\right)$ en cada tramo. Si no cumple, mónínica el diámetro de forma acorde.

D2) Determina el tipo de armario de regulación (Capítulo. 5. Materiales, elementos, accesorios, pp. 5.4-1 a 4).

D3) Determina la dimensión del armario o local de contadores y su superficie mínima de ventilación (Cap. 3. Diseño y construcción, sección 3.3 Construcción de instalaciones receptoras, p. 3.3-20). 


\section{Aplicación y evaluación del CIMA}

Pese a que originalmente el CIMA estaba previsto para su aplicación durante la docencia presencial, la declaración del estado de alarma hizo necesaria su adaptación a la modalidad a distancia. Para ello, se realizó un reajuste del formato de las actividades planteadas para su desarrollo mediante los recursos de la Plataforma Virtual de la Universidad de Sevilla, pudiendo mantenerse los contenidos de las mismas. Sin embargo, este proceso fue bastante costoso y laborioso para mí, teniendo que dedicar muchísimo tiempo a esta tarea, especialmente como consecuencia de mi inexperiencia en el uso de este tipo de herramientas para la docencia online. Esta transición a la docencia a distancia supuso la reducción del número de alumnos que han continuado cursando la asignatura.

Por consiguiente, este CIMA se ha aplicado finalmente a un total de 14 alumnos, de los 26 estudiantes con los que contaba inicialmente. Seguidamente, detallo algunos de los aspectos más relevantes que detecté durante la aplicación del CIMA diseñado:

La Actividad 1 (A1), realizada mediante la herramienta Blackboard Collaborate Ultra, fue muy bien recibida por el alumnado, participando un número considerable de estudiantes en el envío individual de fotografías. Esto permitió generar un debate a distancia bastante interesante a nivel de clase, durante la puesta en común de las imágenes, intercambiándose opiniones muy diversas. Además, esta actividad me facilitó conocer igualmente las ideas previas de los estudiantes, lo que sin duda fue una fuente de información muy valiosa, especialmente de cara a enfocar las sesiones teóricas.

La Actividad 2 (A2) se desarrolló de forma bastante fluida, empleando la herramienta Encuesta de la Plataforma

Ciclos de Mejora en el Aula (2020). Experiencias de Innovación Docente de la US Esta obra se distribuye con la licencia Creative Commons 
Virtual de la Universidad de Sevilla. Pese a que recibí los cuestionarios del total de 14 alumnos, el desarrollo de esta actividad de manera online no me permitió valorar la actitud de los estudiantes durante su cumplimentación (no les pedí que conectasen su cámara por razones de intimidad). Fue una vez analizados los cuestionarios, cuando pude comprobar que no todos los estudiantes realizaron esta actividad con seriedad. El posterior debate del cuestionario en la Actividad 3 (A3), mediante Blackboard Collaborate Ultra, sí que me posibilitó detectar aquellos alumnos que estaban más involucrados y motivados con la tarea, pues se conectaban para realizar más comentarios al respecto. Como en la experiencia del curso previo, los estudiantes se tomaron más en serio la realización del cuestionario final frente al inicial, pese a puntualizar que su realización repercutiría positivamente en la calificación final de la asignatura.

La Actividad 5 (A5), la más importante de este CIMA, fue la más costosa de realizar, como era esperable. La creación de los equipos de trabajo en la Blackboard Collaborate Ultra se llevó más tiempo de lo planificado, debiendo también dedicar unos minutos a explicar el uso de esta herramienta y sus funciones al alumnado, pues también era la primera vez que la usaban. No obstante, hubo una gran implicación por parte de los alumnos, muchos de ellos movidos más por la curiosidad de la actividad, que por el contenido de la unidad que se iba a aprender. Esta actividad supuso para mí una labor de coordinación a gran escala, teniendo que estar pendiente del funcionamiento de cada grupo creado y debiendo ponerme en comunicación con cada uno de ellos por turnos, salvo para aquellas aclaraciones que podrían realizarse a nivel de clase. Para mí fue una experiencia ciertamente estresante, pues como docente tenía la responsabilidad de guiar el proceso y sentía cierta impotencia en mis intentos por gestionar situaciones a distancia que se hubieran podido resolver con

Ciclos de Mejora en el Aula (2020). Experiencias de Innovación Docente de la US Esta obra se distribuye con la licencia Creative Commons 
mayor facilidad de forma presencial. La primera parte de esta actividad me exigió un sobreesfuerzo mental que no tenía previsto. Sin embargo, una vez superadas estas dificultades, pude afrontar mejor las siguientes partes de esta actividad y, los estudiantes, también se sintieron más capaces de interactuar y participar. Finalmente, fue una experiencia singular y de la que me llevo conclusiones bastante positivas.

Las sesiones teóricas (A6) se desarrollaron sin ningún problema destacable en el entorno del Blackboard Collaborate Ultra. En general, los estudiantes participaron de forma activa durante las dos sesiones, respondiendo a las preguntas que les hacía o planteando algunas dudas que se iban aclarando sobre la marcha sin mayor inconveniente. También debo destacar que, de los 14 estudiantes, 6 fueron totalmente inactivos durante estas sesiones y que, en la segunda sesión, solo se conectaron 11 estudiantes.

La Actividad 7 (A7) fue completada solo por 11 estudiantes durante la última sesión, mediante la herramienta Encuesta de la Plataforma Virtual de la Universidad de Sevilla. Por este motivo, esta actividad fue puesta a disposición del alumnado, para que los 3 alumnos restantes que ese día no acudieron a la sesión a distancia, pudieran igualmente completarla. Para ello, se remitió un correo a los estudiantes informando sobre esta actividad. Finalmente, los datos de valoración obtenidos se corresponden al total de 14 alumnos. Durante la sesión, se tuvieron que realizar algunas aclaraciones puntuales de algunas preguntas, no obstante, el CIMA despertó la curiosidad en el alumnado y dio pie a un intenso y bienvenido debate.

Por último, he de destacar que durante la docencia a distancia se registraron problemas técnicos puntuales. Pese a que consiguieron resolverse, algunas de las actividades sufrieron un cierto retraso en su realización por motivos derivados de esos fallos de conexión.

Ciclos de Mejora en el Aula (2020). Experiencias de Innovación Docente de la US Esta obra se distribuye con la licencia Creative Commons 
En la Figura 5 se han comparado los resultados de los cuestionarios inicial y final (A2). Debido al carácter abierto de las preguntas, se ha realizado un análisis empírico y una posterior clasificación de las respuestas de acuerdo a unos escalones de aprendizaje, definidos teniendo en cuenta las recomendaciones de Porlán (2018). Estos escalones pueden identificarse en la Figura 6, en la que se muestra un resumen de los resultados de aprendizaje de los estudiantes.

En general, puede apreciarse que el aprendizaje de los estudiantes ha mejorado un escalón de media, con un reducido número de estudiantes que alcanzan los escalones más altos. Pese a que solo se disponen datos de 14 alumnos, de los resultados de este análisis se han detectado dos aspectos clave: (1) Una dificultad generalizada del alumnado para razonar y justificar sus respuestas, a veces respondiendo aspectos irrelevantes a la pregunta formulada; y, en consecuencia, (2) las respuestas excepcionales y bien justificadas son muy puntuales, limitándose la mayoría de las ocasiones a responder de forma escueta las preguntas o sin razonamientos sólidos.

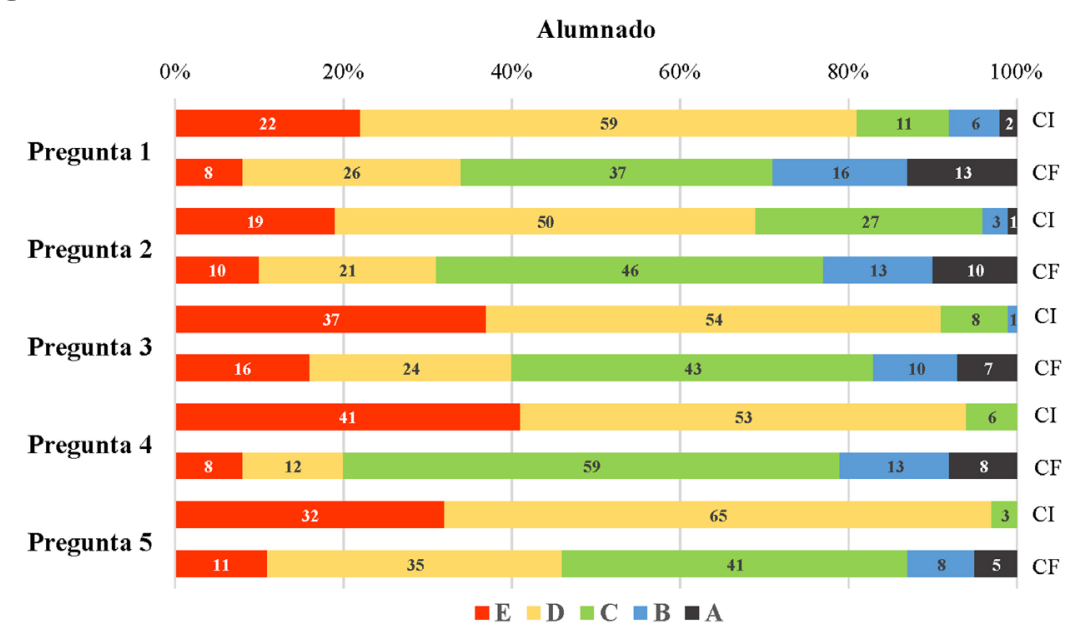

Figura 5. Análisis comparativo de los resultados de los cuestionarios inicial (CI) y final (CF).

Ciclos de Mejora en el Aula (2020). Experiencias de Innovación Docente de la US Esta obra se distribuye con la licencia Creative Commons 


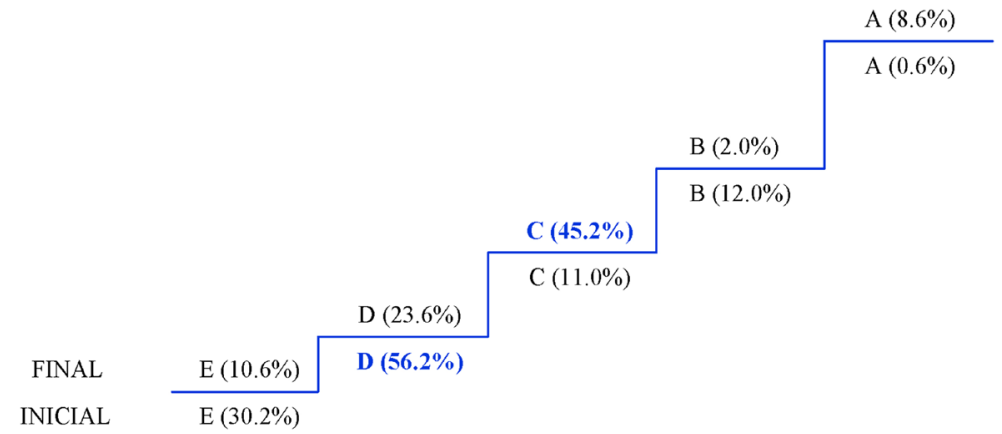

A:Contesta de forma correcta la pregunta y ofrece argumentos sólidos y correctos para justificar su respuesta, basándose en el contenido de las noticias.

B: Contesta de forma correcta la pregunta y ofrece algunos argumentos suficientes y correctos para justificar su respuesta.

C: Contesta de forma correcta la pregunta, pero ofrece argumentos incorrectos e incoherentes para justificar su respuesta.

D: Contesta de forma correcta la pregunta, pero no ofrece argumentos para justificar su respuesta.

E: No contesta lo que se pregunta o deja la respuesta en blanco

Figura 6. Escaleras de aprendizaje inicial y final: evaluación media en la clase.

Pese a las dificultades inherentes que ha supuesto enfrentarme por primera vez como docente a las herramientas y recursos de la enseñanza a distancia, mi valoración personal del CIMA es bastante positiva y satisfactoria. Igualmente, la valoración media de los estudiantes sobre el CIMA y la profesora es muy positiva, como muestra el cuestionario de evaluación que han cumplimentado en la Actividad 7 (A7) (Tabla 4).

Ciclos de Mejora en el Aula (2020). Experiencias de Innovación Docente de la US Esta obra se distribuye con la licencia Creative Commons 
Tabla 4. Cuestionario de evaluación del docente y CIMA. Incluye valoración media del alumno.

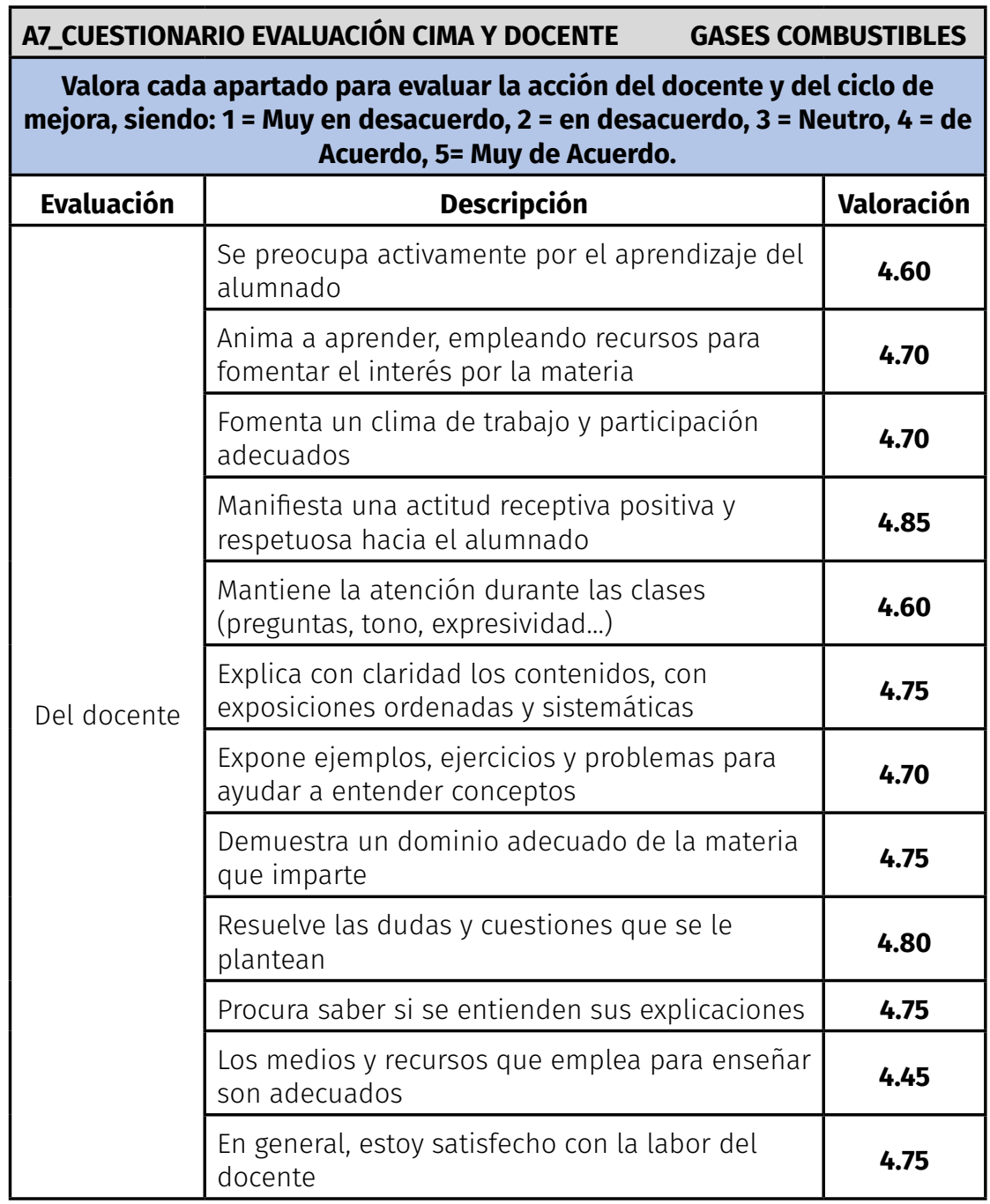

Ciclos de Mejora en el Aula (2020). Experiencias de Innovación Docente de la US Esta obra se distribuye con la licencia Creative Commons Reconocimiento-NoComercial-SinObraDerivada $\quad 4.0$ Internacional (CC BY-NC-ND 4.0.) 


\begin{tabular}{|c|l|c|}
\hline & $\begin{array}{l}\text { Ha sido un planteamiento innovador e } \\
\text { interesante }\end{array}$ & $\mathbf{4 . 1 0}$ \\
\cline { 2 - 4 } Del CIMA & $\begin{array}{l}\text { El cuestionario y las actividades planteadas han } \\
\text { sido útiles para aprender }\end{array}$ & $\mathbf{4 . 4 5}$ \\
\cline { 2 - 4 } & $\begin{array}{l}\text { Los ejercicios planteados han sido apropiados } \\
\text { y sus contenidos claros, estando planificados y } \\
\text { ordenados de forma coherente }\end{array}$ & $\mathbf{4 . 2 5}$ \\
\cline { 2 - 4 } & $\begin{array}{l}\text { La actitud del docente ha sido adecuada, } \\
\text { guiando el proceso, aclarando dudas }\end{array}$ & $\mathbf{4 . 5 5}$ \\
\cline { 2 - 4 } & $\begin{array}{l}\text { El tiempo de trabajo que se le ha dedicado ha } \\
\text { sido apropiado }\end{array}$ & $\mathbf{4 . 3 5}$ \\
\cline { 2 - 4 } & $\begin{array}{l}\text { El enfoque de las actividades ha fomentado mi } \\
\text { interés por aprender }\end{array}$ & $\mathbf{4 . 0 5}$ \\
\cline { 2 - 3 } & $\begin{array}{l}\text { Se deben plantear más actividades de este } \\
\text { estilo }\end{array}$ & $\mathbf{4 . 1 0}$ \\
\cline { 2 - 3 } & $\begin{array}{l}\text { En general, estoy satisfecho con el ciclo de } \\
\text { mejora }\end{array}$ & $\mathbf{4 . 0 0}$ \\
\hline
\end{tabular}

\section{Conclusiones}

Entre las conclusiones y valoraciones personales de este CIMA, hay que destacar que ha sido posible despertar el interés y grado de motivación del alumnado de forma generalizada, ofreciendo una metodología docente alternativa y rompedora frente a las técnicas tradicionales a las que están acostumbrados, siendo muy bien recibida por los estudiantes. Este tipo de actividades ha permitido al alumnado ser consciente de sus propias capacidades y habilidades para resolver problemas reales, llevando a cabo una auto-gestión y auto-evaluación más directa del proceso de aprendizaje. Además, ha quedado patente la mejora de los resultados académicos del alumnado, como muestran los resultados de los cuestionarios. Igualmente, el trabajo en equipos cooperativos y colaborativos ha favorecido el intercambio de ideas y opiniones entre el alumnado, generando una interdependencia social bastante positiva. Ello, sumado a la labor de investigación que han realizado los estudiantes, tanto de forma individual como grupal, ha 
permitido ceder el protagonismo al alumnado durante su proceso de aprendizaje, lo que, al mismo tiempo, refuerza su ambición e interés. También hay que resaltar que se han elaborado recursos materiales y digitales que han sido empleados por el alumnado como material de estudio y que pueden ponerse a disposición de futuros estudiantes en próximos cursos.

A pesar de los buenos resultados, los estudiantes han reconocido las dificultades de realizar tareas en equipos durante la docencia a distancia, por motivos de coordinación con herramientas telemáticas. De hecho, muchos han transmitido su preferencia a realizar tareas de carácter individual durante una docencia no presencial.

Pese a ello, un alto porcentaje de alumnos han valorado positivamente la implementación de este tipo de actividades innovadoras en la materia y han defendido la necesidad de seguir incorporándolas en el futuro a otros temas. Por ello, veo muy positivo extender esta metodología de trabajo a otras unidades temáticas para el próximo curso y, como mejora ante una posible docencia mixta, incorporar apartados de trabajo carácter individual como grupal.

Ciclos de Mejora en el Aula (2020). Experiencias de Innovación Docente de la US Esta obra se distribuye con la licencia Creative Commons 
Palabras clave: Acondicionamiento e instalaciones, grado en fundamentos de arquitectura, docencia universitaria; experimentación docente universitaria.

Keywords: Refurbishment and installations; degree in architecture fundamentals; university teaching; university teaching experimentation.

\title{
Referencias bibliográficas
}

\begin{abstract}
Ausubel, D., Novak, J. Y. H. H., \& Hanesian, H. (1976). Significado y aprendizaje significativo. En Psicología educativa: un punto de vista cognoscitivo (pp. 53-106). México: Trillas.
\end{abstract}

Bruner, J. (2011). Aprendizaje por descubrimiento. NYE U: Iberia.

Calama-González, C.M. (2020). Unir teoría y práctica a través de lo virtual: una innovación docente aplicada al diseño de instalaciones. En Ciclos de mejora en el aula. Año 2019. Experiencias de innovación docente de la Universidad de Sevilla (pp. 336-35). Sevilla: Universidad de Sevilla. ISBN 978-84-472-2191-2.

Gagné, R. M. (1970). Las condiciones del aprendizaje. Madrid: Aguilar.

Guilar, M. E. (2009). Las ideas de Bruner: de la revolución cognitiva a la revolución cultural. Educere, 13(44), pp. 235-241.

Piaget, J. (1981). La Teoría de Piaget. Monografías de infancia $y$ aprendizaje. Journal for the Study of Education and Development, 4, pp. 124-125.

Porlán, R. (2017). Enseñanza universitaria. Cómo mejorarla. Madrid:Morata.

Vygotsky, L. S., \& Souberman, E. (2012). El desarrollo de los procesos psicológicos superiores. Barcelona: Crítica.

Ciclos de Mejora en el Aula (2020). Experiencias de Innovación Docente de la US Esta obra se distribuye con la licencia Creative Commons 